A PROJECTION PHONEIDOSCOPE

By Geo. H. Stone, Kent's Hill, Me.

Br combining the essential features of the phoneidoscope 122) with President Morton's method of projecting interfer. ence tints, the sound vibration figures of soap films can
easily be projected and even measured on the screen before an audience. The method has also this advantage, that the
movements in the whole film can be observed at once. A movements in the whole film can be observed at once. A
bright light is needed, preferably from the sun or electric reflected beam is passed through a convex lens. A better reflected beam is passed through a convex lens. A better horizontal, reflection from mirrors is needed, which causes inches in diameter are so strong that they become monochromatic before bursting, and last longer when in vibration
than when still. Under the action of sound pulsations the than when still. Under the action of sound pulsations the
film becomes violently agitated, rapid vortical motions enfilm becomes violently agitated, rapid vortical motions en
sue, polygonal figures bounded by bright lines appear, and
often in the center of each is a often in the center of each is a whirling spot of light, connected with the perimeter of the polygon by faint vortical or polygons, while a square film has a decided tendency to
break into nearly regular squares, and a hexagonal film into hexagons. The interference colors are very brilliant, and the whole forms an instructive and beautiful experiment,
the figures varying with every change in the pitch or quality the voice or sounding body.

THE COMING TOTAL SOLAR ECLIPSE. By J. Norman Lockyer.

THERE is no doubt whatever that the eclipse which will
weep over the United States next July will be observed a no eclipse has ever been observed before. The wealth of men, the wealth of instruments, and the wealth of skill in all matters astronomical already accumulated there, make to ourselves what the golden age will be like there, when already they are so far ahead of us in so many particulars. Draper, Hall, Harkness, Holden, Langley, Newcomb, Pe-
ters, Peirce, Pickering, Rutherfurd, Trouvelot, and last, but ters, Peirce, Pickering, Rutherfurd, Trouvelot, and last, but pansion with a little thought, when one thinks of the men who will be there. One knows, too, that all the enthusiasm of devoted students and all the appliances of modern science have borne so noble a part-will not be lacking. So that we
may be sure that not only all old methods but all possible may be sure that not only all old methods but all possible
new ones will be tried to make this year one destined to be new ones will be tried to make this year one destined to be
memorable in the annals of science side by side with 1706 ,
1851,1860 , and other later

Thank heaven, too, there is no necessity that the thankless task of organizing an " Eclipse Expedition" from this other reasons because - and this is a very hopeful sign of inIsmay, Imray \& Co.. the owners of the White Star Line English observers by a considerable reduction of fares, and the readers of Nature have already been made aware, have done the like in the case of observers coming from Europe in their individual capacity. ${ }^{*}$

The progress in that branch of knowledge which requires
the aid of eclipse observations has been so rapid during the the aid of eclipse observations has been so rapid during the last few years, that the eclipse of 1868, though it happened
only ten years ago, seems to be as far removed from the present as the Middle Ages are in regard to many other since that year, when in the hands of Janssen, Pogson, Herschel, and others it added so enormously to our knowledge, has gradually covered larger and larger ground, and each
successive eclipse in $1869,1870,1871$, and 1875 has seen some variations in its use, so that its employment has proved
most novel, if not the most powerful, side of the attack. Young's work of 1869 will no doubt form the keynote much that will be done this year so far as the coronal at-
mosphere is concerned. It will be remembered that Young in 1869 observed a continuous spectrum, while Janssen in presence of the more prominent Fraünhofer lines, notably
D. This positive observation from so distinguished an obperver demands attention, not only on its own account, but because of the question which hangs upon it, which is this:
Does the corona reflect solar light to us or does it not, and if it does, where are those particles which thus act as reflectare silent, as the method employed was not intended to discriminate between a continuous and a discontinuous spectrum. " 1474 ," is more familiar to us now; and yet there has been "1474," is more familiar to us now; and yet there has been with regard to this line above all others, there appears to be due to iron at all; with the most powerful arc there is no iron line to be seen there. Then Secchi attributed it to hydrogen, though I am not aware on what evidence. But,
whatever be its origin, the fact remains that we now know by its means that the solar hydrogen is traversed and enwrapped by the substance which gives rise to the line to an enormous height, so that it forms the highest portion of the
atmosphere which is hot enough to render its presence mani-
fest to us by spectral lines. Here, so far as I know, only fest to us by spectral lines. Here, so far as I know, only
one point of difference remains. In 1871 I most distinctly saw the line trumpet-shaped, that is, with the base broadenJangsen saw it stopping short of the spectrum of the photosphere. The importance of this point is that, supposing
one of us to be mistaken, and one or other observation to represent a constant condition, then, if the line broadens
downward till the sun is reached, we are dealing with a gas lighter than hydrogen, capable of existing at a high temperature, which thins out as the other gases and vapors do, in consequence of its vapor density being below that of hydro-
stant condition, it represent, if the line stops short as a condissociated at the lower levels, and is therefore probably a

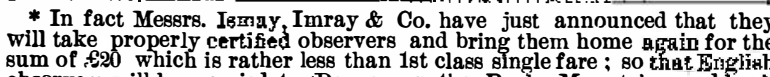
sum of f20 which is rather less than 1st class single fare; so thit Finglizh
observers will be carried to Denver or the Rocky Mountains and bsch
again for the sum of $£ 34$. compound gas; and then the question arises whether it has
not hydrogen as one of its constituents. Perhaps I may conveniently refer to a paper of mine which Perhaps I may conveniently refer to a paper of mine which
was lately read at the Royal Society in this connection, beclosest study at the present time are precisely these higher
reaches of the sun's atmosphere. There is little doubt, I reaches of the sun's atmosphere. There is little doubt,
think, that around the sun's visible atmosphere matter ex ists at a temperature low enough not to give us its autobi
ography in the bright line manner, and there is evidence that matter existing under such conditions, absorbing as it must us an absorption of the fluted kind, or again will absorb only the blue or ultra-violet region.

Now the more the chemistry of the reversing lower laye
of the sun's atmosphere-that in which the upper level the photosphere is bathed-is examined, the more metallic is it found to be. For instance, my own work has enabled but of metalloids in this region I have traced none. Th persistency with which metal after metal revealed itself to some time ago that perhaps the metalloids lay as a whole
above the metals, and shortly afterward I obtained evidence above the metals, and shortly afterward I obtained evidence rendered probable by fluted bands, and not by lines. There fore the matter could be considered to be placed beyond all doubt. present in the spectrum of the electric arc, as photographed, which bands vary very considerably in strength according bands of carbon-a point denied by Angström and Thalèn.
This point I have settled by two photographs, in which hat of carbon in air, the other of carbon in dry chlorine. The next point was to insure accuracy by the most posibon bands. Such a shift is produced when the part of the
arc photographed,is not perfectly in the prolongation of the arc photographed,is not perfectly in the prolongation of the
axis of the collimator of the spectroscope. Its effect is to throw the lines of iron, for instance, a little to the right or a
little to the left of the Fraunhofer lines with which they
really correspond. I have now obtained a photngraph which supplies such evidence. There are metallic lines close to the carbon bands
which are prolongations of Fraünhofer's lines, while the lines which I have already mapped at $W$. L., $39 \cdot 27$ and
$39 \cdot 295$, in the spectrum of iron, are also absolute prolongations. Therefore there is no shift in the carbon flutings, and
the individual members of the fluted spectra in the brightest portion are absolute prolongation

Now how does this connect itself with observations of the upper parts of the solar atmosphere?

Angström has already shown that the true carbon lines
which we get when a coil and jar are employed are not reversed in the spectrum of the sun, and I have alread not $r$ cow that the calcium spectrum in the sun is similar to the spectrum obtained when the spark, and not the arc, is employed.
Accompanying the change from a high to a higher temperature there is a change in the intensity of the lines--some
thicken, others become thinner. We can only match the very powerful coil and jar-so powerful, indeed, that the very powerful coil and jar - so powerful, indeed, that the
lines, and not the flutings, of carbon would be visible in the
spark given by it. It is fair, then, to say that, if carbon spark given by it. It is fair, then, to say that, if carbon
were present with the calcium in the sun's reversing layer, we appearing as they do.

As we do not get this evidence, we are driven to the con-
clusion that the carbon vapor exists not only in a more complicated molecular condition (as is evinced by the flutings) plicat the metallic vapors in the sun's atmosphere, but at a
lower temperature. It must, therefore, exist above the chromosphere, that is, in a region of lower temperature. Lower pressure, again, is indicated by the feeble reversal, so that
everything points to a high level. The question is, will this region be recognized during the
The

Coming down lower we reach a level better known, and of which; perhaps, the interest during the eclipse will now photography. One good photograph of the lines visible in
the lower cromosphere will be of incalculable value tempts may be made on the cusps just before and after totality, and if only one of these succeeds we shall have the
ordinary solar spectrum as a scale. If good pictures near region to enable us to determine the chemical origin of the bright lines photographed. These remarks apply to attempts made with spectroscopes furnished with slits in the ordinary for the Siam eclipse in 1875, the method suggested by Prof.
Young and myself for the Indian eclipse of 1871, will also Young and myself for the Indian eclipse of 1871 , will also
be taken advantage of; here the cromosphere itself becomes
the slit. A dispersed series of spectral images of the thing the slit. A dispersed series of spectral images of the thing focused on a slit, is obtained, the position of each image in
the spectrum enabling its chemical origin to be ascertained if only a comparison spectrum can be secured at the same

In 1875, in the expedition to Siam, the photographs of this
nature were obtained by means of a prism, and the results nature were obtained by means of a prism, and the results
obtained by that expedition led me to think that, possibly, obtained by that expedition led me to think that, possibly, prit might be opplied under very favorable conditions if the prism, or train of prisms, hitherto employed, were replaced
by a reflection grating, with which the generosity of Mr. Rutherfurd has made many of us familiar for the simple reason that, while a prism only gives us one spectrum, a of the line, perpendicular to its surface. Of these two or hree are bright enough to be utilized on each side, so that To test this notion I made the following experiment with grating given to me by Mr. Rutherfurd. This magnificent instrument contains 17,280 lines to the inch, ruled on glass, In front of the condenser of an electric lamp adjusted to * These are strontium, lead, cadmium, potassium, cerium, uranium
vanadium, palladium, molybdenum, indium, lithium, rubidium, coesium, cardboard, forming a ring some 2 inches in interior diameintended to represent the chromosphere, and formed my ar-
ing At some distance from the lamp I mounted a $33 / 4$ inch
ificial eclipse. At some distance Srome distance short of the focus I placed
the grating; the spectrum of the circular slit, illuminated the grating; the spectrum of the circular slit, illuminated
by sodium vapor and carbon vapor, was photographed for
the first, second, and third orders on one side. The third order spectrum, showing the exquisite rings due to the car-
bon vapor flutings, was produced in forty-two seconds. The first order spectrum, obtained in the same period of time, expecting too much that we should be able to take a photograph of the eclipse, in the third order, in two minutes. Similarly we may hope for a photograph of the second order
in two minutes, and it is, I think, highly probable also that in two minutes, and it is, I think, highly probable also that
a photograph of the first order may be obtained in one minphotograph of the first order may be obtained in one min-
ute. To make assurance doubly sure, the whole of the toute. To make assurance doubly sure, the whole of the to-
tality may be used during the coming eclipse, but if there
be several such attempts made, it will certainly be worth while to trywhat a shorter exposure will do. Now by mounting photographic plates on both sides of
he axis, one solidly mounted equatorial of short focal length may enable us to obtain several such photographs, with va-
rying lengths of exposure. I insist upon the solidity of the rying lengths of exposure. I insist upon the solidity of the mounting, because, if any one plate is to be exposed during
the whole of totality, the instrument must not be violently however, it is quite possible to obtain more than one photohowever, it is quite possible to obtain more than one photo-
graph of the lower order spectra without any such disturbance in this way. The same plate may be made to record
bree or even four exposures in the case of the first order in an eclipse of four minutes' duration, by merely raising or other equivalent contrivance, so that a fresh portion of the the spectra of the second order are to be recorded may be

If one equatorial thus mounted were to be devoted to each quadrant of the coronal atmosphere, it is certain, I think,
that most important results would be obtained. -Nature.

\section{ON THE AEROLITIC EPOCH OF NOVEMBER}

By Prof. Daniel Kirkwood.

IT is now well known that clusters of small meteors-the so-called shooting stars-move in elliptic orbits about the
sun. Catalogues of fire-balls and meteoric stones indicate, moreover, that groups of larger bodies, somewhat widely distem, their orbits in certain cases intersecting that of the epochs, the date being nearly coincident with that of the cently supposed the meteorites of this epoch to revolve in the same orbit with the nebulous swarm which furnished the
showers of 1833,1866 and $1867 . \dagger$ Later study of the facts, however, has rendered the theory of this intimate relation
extremely improbable. The principal phenomena of this epoch (not including star showers) are the following:

(a.) 1582, meteoric phenomena at Zurich.

(b.) 1765, an extraordinary meteor at Frankfort.

(c.) $\left\{\begin{array}{l}1820, \text { a detonating meteor seen in Russia. } \\ 1822 \text {, fall of aerolites at Potsdam and Lei }\end{array}\right.$

(e.) 1828, a great meteor seen in full sunshine in France.

$\left(\begin{array}{l}(f .) \\ (g .) \\ 1849, \text { a a fall of aerolites in France. }\end{array}\right.$

(h.) 1849, a large meteor seen in Mecklenberg.

(i.) 1856, a metoric stone fell in Italy

$(j$.$) 1877, a brilliant meteor$
ther in Wisconsin

REMARKS.

(a.) This so-called "fall of fire from heaven" occurred on
he 28 th of October, O. S., or November 7th, N. S. Making the 28th of October, O. S., or November 7th, N. S. Making
allowance for the precession of the equinoxes, the date cor(b.) This bolide was observed November 11th, and is the ouly one in our list which occurred very near the epoch of (c.) See Greg's catalogue of fire-balls and meteoric stones;

(d.) Several aerolites fell at this date near Potsdam, and (e.) See Quetelet's catalogue

$(f$.) This fall of aerolites occurred on the evening of November 13th, in the department de l'Ain, France. The me-
teor was unconformable to the radiant of the Leonids, its mothe collection of Prof. Shepard, of A mht. A fragment is in (g.) The meteoric phenomena of this date are thus described naried in color; a bright cloud visible one and a half hours
Varione after; according to some a detonation heard fifteen minutes after bursting. Seen also like a stream of fire between them in the town of Tripoli itself."

(h.) This fire-ball appeared on the same evening or night. Greg's catalogue.

(i.) This aerolite fell at Trezano. A fragment is in the
collection of Prof. Shepard. (j.) A large meteor was seen by Professor Robert C. Hindley, of Racine, Wisconsin, on sunday evening, November meteor is thus described by Professor H. in the ScIENTIFIC
AMERICAN for December 1, 1877: "Direction N. N. E.; altifude at commencement of course about $30^{\circ}$; length of course
from $10^{\circ}$ to $12^{\circ}$; time of falling about 8 seconds. It fell toward the west, making an angle in falling to the earth of about $65^{\circ}$ with the vertical passing through the body. During the latter three-fourths of its course, its length, includnucleus was very brilliant; its color at first a yellowishwhite, then a light grcen, and lastly, a greenish-yellow.
Could its color have been due to boron, thallium, etc.? I and no record in any of the numerous analyses of meteoric
tones of the presence of elements likely to give the green color."

On the following evening; November 12 th, at $6 \mathrm{~h} 26 \mathrm{~m}$. * Read before the American Philosophical Society, March 1, 187\% t A list of stone-falls, detonating meteors and large fre-balls which
have appeared abuut thisepoch is given in Meteoric Astronomy, pp. 58-60. 